\title{
Special issue on user profiling and behavior adaptation for human-robot interaction
}

The idea of making a special issue on User Profiling and Behavior Adaptation for Human-Robot Interaction came to our minds during the organization of the BAILAR workshop (Behavior Adaptation, Interaction and Learning for Assistive Robotics) held in conjunction with RO-MAN 2016. The aim of the workshop was to bring together the works of many experts in this multidisciplinary subject that involves different competencies and knowledge which span from pattern recognition and machine learning to user profiling and to robot behavior control. Creating robotic systems capable of correctly modeling and recognizing the human behavior and of adapting their behaviors to the user is a very critical and open task. In this direction, the goal of this special issue is to highlight the advances from a wide-angle perspective on the topics, as well as to further stimulate excellent fundamental and applied research.

This special issue contains 12 contributions. The first contribution [8] is a survey that introduces the research topics addressed in this context by providing some pointers to the recent literature. Moreover, the authors provided a classification of the considered works with respect to three different viewpoints, namely from the physical, the cognitive, and the social aspects of the interaction both in the case of user profiling and of behavioral adaptation.

According to Rossi et al. [8], one of the required characteristics for a socially interactive robot should be to perceive, learn, and recognize models of the other agents, with which it is interacting. Existing studies on human activity recognition typically follow a pattern recognition approach to the users' movements in the space. Such approaches extract different information/features from the sensor data and use machine learning algorithms, typically classification methods, to identify activity patterns. A large number of different approaches has been developed in the literature. This is reflected in this issue with five articles on this topic accepted. In particular, the works of Luvizon et al. [5] and Nunes et al. [7] deal with the problem of feature extraction for action and activity recognition. In particular, Luvizon et al. [5] propose to extract sets of spatial and temporal local features from subgroups of joints identified by using an RGB-D camera. Local features are aggregated into several feature vectors, so providing a good representation of long and short actions. All the feature vectors are then combined by a metric learning method used to extract the most discriminant information from features. Some of the challenges in this field are related to the ability to perform the recognition in real-time, to the correct segmentation of the input in case of continuous gesture recognition, and to recognize gesture patterns with incomplete data. In [7], a different feature extraction technique is proposed that is intended to rely on few training examples evidencing fast training times for real-time applications. In this case, the feature extraction stage is based on key poses. Each action window is delimited by two consecutive and automatically identified key poses, where static (i.e. geometrical) and max-min dynamic (i.e. temporal) features are extracted. These features are used to train a random forest classifier. Simao et al. [11] introduce an approach to perform the recognition of time series segments, targeted at natural language processing of hand gestures. The work deals with the problem of data dimensionality reduction for gesture recognition to allow to represent such data in a lowdimensional space, making the classification process more efficient. This approach has the advantage of allowing accurate classification to be performed with partial (incomplete) gesture data. Yang et al. [12] deal with the problem of simultaneous gesture segmentation and recognition and propose a continuous hand gesture recognition method based on trajectory shape information. A gesture trajectory is divided into a set of key frames. Variable-sized trajectory segments are then generated using the selected key frames by using a Convolutional Neural Network architecture suitable for totaltrajectory-shape recognition. For recognition, these trajectory segments are fused and examined to determine whether the segment belongs to a class among intended gestures or a non-gesture class based on the fusion of shape information and temporal features. Finally, De Rosa et al. [2] deal with the problem of recognition human activities from streaming sources with a nonparametric approach, i.e., their structure should adapt in response to the incoming data, by incrementally learning a model which adaptively covers the feature space with simple and local classifiers, and by employing an active learning strategy to reduce annotation requests.

When dealing with modeling and learning of the user preferences with respect to trajectories and tasks, the typical approaches rely on the so-called learning by demonstration methods, where the user teaches the robot by providing examples. For example, Mokhtari et al. [6] analyze the problem of learning robot task models in the case of loops. Such loops may correspond, for example, to a sequence of actions that is successively applied to different objects. This work presents a model to detect and represent loops of actions in a task demonstration and to adapt and exploit loops to different instances of the same class of tasks. While in [6], in order to conceptualize the task, the robot is provided with step-by-step operating instructions, indirect teaching can also be achieved by letting the robot relying only on user feedback. In this issue, two contributions deal with learning processes that rely on the user 
feedback. The first one is in the domain of the development of exoskeleton robots that physically assist humans [4]. In this work, the authors explore a data-driven learning approach for designing assistive strategies from the user-robot physical interaction. The learning problem is formulated as a policy search problem and exploits a data-efficient model-based reinforcement learning framework. Instead of explicitly providing the desired trajectories in the cost function, the considered cost function only considers the users muscular effort measured by electromyography signals (EMGs) to learn the assistive strategies. The second one [10] addresses the problem of learning strategies for exploring the environment. In particular, the authors investigate how the state space exploration can be optimized by letting a human expert guide the robot during its learning. This paper presents a novel method which combines Reinforcement Learning and Supervised Progressively Autonomous Robot Competencies (SPARC). By allowing the user to fully control the robot and by treating rewards as implicit, SPARC aims to learn an action policy while maintaining human supervisory oversight of the robots behavior.

For robots to successfully take part in social interactions with people, they must be capable of recognizing, interpreting not only the intended communication and physical interaction, but also the social cues displayed by a human. Hence, the effective recognition of such cues can be used to improve the interaction between the human and a robot. In the context of a robot providing recommendation for fashion apparels, the work of De Carolis et al. [1] present a multi-modal framework for recognizing the attitude of the user during the conversation. Speech prosody, body poses, and facial expressions have been taken into account for providing implicit feedback to the system and for refining the recommendation accordingly. The proposed model for the recognition of the user emotional feedback has been represented as a Dynamic Bayesian Network, due to the ability of this formalism to represent the user mental state by taking into account uncertainty and graduality.

All the previously mentioned contributions dealt mainly with user profiling and recognition, however, in order to be effective, a robot should also be able to modify and adapt its behavior accordingly. Indeed, for improved and natural human-robot cooperation, human users will learn how to interact with the robot but, at the same time, the robotic systems should adapt to the users. The recognition of the user's status and gestures is used in [9], where the authors present a real-time approach for human-aware motion re-planning using a two-level hierarchical architecture. The lower level leverages stable dynamical systems to generate motor commands and to on-line reshape the robot trajectories. The reshaping strategy modifies the velocity of the robot to increase the human safety in case of close interaction with the robot, to guarantee the correct task execution in case of unforeseen obstacles (including the human), and to re-plan on-line the current task taking into account the human behavior. The higher level of the architecture monitors the human and provides to the lower level information about the human status.

Finally, in the last contribution [3], Gomez-Donoso et al. developed a multi-sensor system for rehabilitation and interaction with persons with motor and cognitive disabilities. The system enables them to perform different therapies using multiple modes of interaction (head and body pose, hand gestures, voice, touch, and gaze) depending on the type and degree of disability. Through a training process, the system can be customized enabling the definition of the patients own gestures for each sensor. The main aim of the study was to understand the usability/accessibility of the system.

We sincerely thank all the authors for submitting their work to the special issue, the reviewers for their comments and suggestions, and the PRLetters editorial team for their support and guidance.

Silvia Rossi*

Department of Electrical Engineering and Information Technologies, University of Naples Federico II, via Claudio, 80125 Naples, Italy

Dongheui Lee Department of Electrical and Computer Engineering, Technical University of Munich, Munich, Germany

*Corresponding author. E-mail addresses: silvia.rossi@unina.it (S. Rossi), dhlee@tum.de

(D. Lee)

\section{References}

[1] B. De Carolis, M. de Gemmis, P. Lops, G. Palestra, Recognizing users feedback from non-verbal communicative acts in conversational recommender systems, Pattern Recognit. Lett. (2017). (this issue)

[2] R. De Rosa, I. Gori, F. Cuzzolin, N. Cesa-Bianchi, Active incremental recognition of human activities in a streaming context, Pattern Recognit. Lett. (2017). (this issue)

[3] F. Gomez-Donoso, S. Orts-Escolano, A. Garcia-Garcia, J. Garcia-Rodriguez, J.A. Castro-Vargas, S. Ovidiu-Oprea, M. Cazorla, A robotic platform for customized and interactive rehabilitation of persons with disabilities, Pattern Recognit. Lett. (2017). (this issue)

[4] M. Hamaya, T. Matsubara, T. Noda, T. Teramae, J. Morimoto, Learning assistive strategies for exoskeleton robots from user-robot physical interaction, Pattern Recognit. Lett. (2017). (this issue)

[5] D.C. Luvizon, H. Tabia, D. Picard, Learning features combination for human action recognition from skeleton sequences, Pattern Recognit. Lett. (2017). (this issue)

[6] V. Mokhtari, L.S. Lopesa, A.J. Pinhoa, Learning robot tasks with loops from experiences to enhance robot adaptability, Pattern Recognit. Lett. (2017). (this issue)

[7] U.M. Nunes, D.R. Faria, P. Peixoto, A human activity recognition framework using max-min features and key poses with differential evolution random forests classifier, Pattern Recognit. Lett. (2017). (this issue)

[8] S. Rossi, F. Ferland, A. Tapus, User profiling and behavioral adaptation for hri: a survey, Pattern Recognit. Lett. (2017). (this issue)

[9] M. Saveriano, F. Hirt, D. Lee, Human-aware motion reshaping using dynamical systems, Pattern Recognit. Lett. (2017). (this issue)

[10] E. Senft, P. Baxter, J. Kennedy, S. Lemaignan, T. Belpaeme, Supervised autonomy for online learning in human-robot interaction, Pattern Recognit. Lett. (2017) (this issue)

[11] M. Simao, P. Neto, O. Gibaru, Using data dimensionality reduction for recognition of incomplete dynamic gestures, Pattern Recognit. Lett. (2017). (this issue)

[12] C. Yang, D.K. Han, H. Ko, Continuous hand gesture recognition based on trajectory shape information, Pattern Recognit. Lett. (2017). (this issue) 